\title{
Circular RNAs as potential biomarkers and therapeutics for cardiovascular disease
}

\author{
Weitie Wang ${ }^{1}$, Yong Wang ${ }^{1}$, Hulin Piao ${ }^{1}$, Bo Li $^{1}{ }^{1}$, Maoxun Huang ${ }^{1}$, Zhicheng Zhu ${ }^{1}$, Dan Li ${ }^{1}$, Tiance Wang ${ }^{1}$, Rihao \\ Xu ${ }^{1}$, Kexiang Liu ${ }^{\text {Corresp. } 2}$ \\ ${ }^{1}$ Department of Cardiovascular Surgery, Department of Cardiovascular Surgery of the Second Hospital of Jilin University, Jilin, China \\ 2 Department of Cardiovascular Surgery, Department of Cardiovascular Surgery of the Second Hospital of Jilin University, Jilin, china \\ Corresponding Author: Kexiang Liu \\ Email address: kxliu64@hotmail.com
}

Circular RNAs (circRNAs) are genetic regulators that were earlier considered as "junk." In contrast to linear RNAs, they have covalently linked ends with no poly-adenylated tails. CircRNAs can act as RNA-binding proteins, sequestering agents, transcriptional regulators, as well as microRNA sponges. In addition, it is reported that some selected circRNAs are transformed into functional proteins. These RNA molecules always circularize through covalent bonds, and their presence has been demonstrated across species. They are usually abundant and stable as well as evolutionarily conserved in tissues(liver, lung, stomach), saliva, exosomes, and blood. Therefore, they have been proposed as the "next big thing" in molecular biomarkers for several diseases, particularly in cancer. Recently, circRNAs have been investigated in cardiovascular diseases (CVD) and reported to play important roles in heart failure, coronary artery disease, and myocardial infarction. Here, we review the recent literature and discuss the impact and the diagnostic and prognostic values of circRNAs in CVD. 


\section{Circular RNAs as potential biomarkers and therapeutics 3 for cardiovascular disease}

6 Weitie Wang, Yong Wang, Hulin Piao, Bo Li, Maoxun Huang, Zhicheng Zhu, Dan Li, Tiance

7 Wang, Rihao Xu, Kexiang Liu

8

9 Department of Cardiovascular Surgery of the Second Hospital of Jilin University, Ziqiang Street 10 218, Changchun, Jilin 130041, China

Corresponding Author:

Kexiang Liu, M.D. P.H.D., Department of Cardiovascular Surgery of the Second Hospital of Jilin University, Ziqiang Street 218, Changchun, Jilin 130041, China. Tel.: 011-86 43181136791, Fax: 011-86 431-81136791, E-mail address: kxliu64@hotmail.com.

\section{Abstract}

Circular RNAs (circRNAs) are genetic regulators that were earlier considered as "junk." In contrast to linear RNAs, they have covalently linked ends with no poly-adenylated tails. CircRNAs can act as RNA-binding proteins, sequestering agents, transcriptional regulators, as well as microRNA sponges. In addition, it is reported that some selected circRNAs are transformed into functional proteins. These RNA molecules always circularize through covalent bonds, and their presence has been demonstrated across species. They are usually abundant and stable as well as evolutionarily conserved in tissues (liver, lung, stomach), saliva, exosomes, and 
27 blood. Therefore, they have been proposed as the "next big thing" in molecular biomarkers for 28 several diseases, particularly in cancer. Recently, circRNAs have been investigated in cardiovascular diseases (CVD) and reported to play important roles in heart failure, coronary artery disease, and myocardial infarction. Here, we review the recent literature and discuss the impact and the diagnostic and prognostic values of circRNAs in CVD.

\section{BASIC REPORTING}

\section{Introduction}

Almost $70 \%$ of the human genome is transcribed. However, protein-coding genes account for only $1 \sim 2 \%$ of the human genome, whereas the majority of transcripts are noncoding RNAs (ncRNAs) (Djebali et al., 2012) .ncRNA can be divided into two types: housekeeping ncRNAs and regulatory ncRNAs ${ }^{+}$. The latter consists of microRNAs (miRNAs, $8.13 \%$ ), small nuclear RNAs (snRNAs, 8.21\%), small interfering RNA (siRNAs, 4.07\%), and long noncoding RNAs (lncRNAs, 69.42\%) (Frankish et al., 2018) (Figure 1). ncRNAs can also be categorized into two families based on size: large (> $200 \mathrm{nt})$ or small ( $<200 \mathrm{nt}$ ) (Taft et al., 2010). Between the two - families, the large ncRNAs are produced by the same transcription and splicing machinery as mRNA, whereas small RNAs are always transcribed from genomic loci and processed by specific nucleases. Circular RNAs (circRNAs) are a type of ncRNA that are abundant and stably exist in living organisms. They are different from linear RNAs because of their covalently closed-loop structure, which makes them gene expression regulators, and have potential clinical therapeutic values (Fan et al., 2017).

With the discovery that ncRNAs are necessary for heart physiology, the important roles of these molecules in cardiovascular disease (CVD) has become evident (Barwari, Joshi \& Mayr, 2016). The main groups of ncRNAs, including miRNAs, lncRNAs, and circRNAs have been reported to be relevant for cardiovascular physiology and disease (Fan et al., 2017; Greco, Gorospe \& Martelli, 2015; Wojciechowska, Braniewska \& Kozar-Kamińska, 2017). Particularly, in the past several years, miRNAs and lncRNAs were shown to be critical contributors to cardiovascular pathophysiology. Moreover, they were reported to be potential biomarkers for several diseases, as they were abundant and stable as well as evolutionally conserved in tissue 
56 (liver, lung, stomach), saliva, exosomes, and blood (Wang, Chen \& Sen, 2016; Yang et al., 2017;

57 Shi \& Yang, 2016; Li et al., 2016)

58

59

60

61

62

63

64

65

66

67

68

69

70

71

72

73

74

75

76

77

78

79

80

81

82

83

84

85

CVDs are a major cause of death worldwide. Early diagnosis and treatment can reduce major adverse cardiovascular events (MACE) (Kim et al., 2011). Thus, efficient biomarkers are important for diagnosis of CVD especially in cardiac injury. Cardiac troponins T/I (cTnT/I) (Heil $\&$ Tang, 2015) and creatine kinase myocardial band isoenzyme (CK-MB) have been widely used as biomarkers for myocardial infarction (MI), while brain natriuretic peptide (BNP) has been used for heart failure (HF) for a long time since long (Calzetta et al., 2016). Ideal biomarkers must be sensitive and specific for specific diseases and should not be influenced by heterogeneity of the heart-associated disease, lifestyle, patient's age, and genetic background (Kondkar \& AbuAmero, 2015; Enroth et al., 2014). Currently, highly sensitive biomarkers are urgently needed for earlier diagnosis of CVDs.

Cardiac physiology is a complex balance between electrical stimuli and chemicals related to the mechanism of contraction. Recently, ncRNAs were reported to be ideal regulators of the cardiovascular system, and circRNAs were linked to CVD (Thum \& Condorelli, 2015; Uchida \& Dimmeler, 2015; Vegter et al., 2016). CircRNAs are a class of ncRNAs that are more stable than linear RNAs (Suzuki \& Tsukahara, 2014), as they form a covalently (Wilusz \& Sharp, 2013) closed continuous loop, which is resistant to RNase R activity (Gao, Wang \& Zhao, 2015). They were thought to be aberrant RNA splicing products with no functions (Sanger et al., 1976) for nearly two decades until bioinformatics methods and RNA sequencing (RNA-Seq) technologies indicated their association with specific biological processes (Salzman et al., 2012) These circRNAs are always stored in the cytoplasm (Jeck et al., 2013) and only a small part exists in the nucleus (Ebbesen, Hansen \& Kjems, 2017). Many studies have found that they participate in cell proliferation (Zhao et al., 2015), migration and invasion (Zhong, Lv \& Chen, 2016; Ng et al., 2016; Wang et al., 2017; Xu et al., 2017), gene transcription (Memczak et al., 2013), and function as gene expression regulators, miRNA sponges (Memczak et al., 2013; Hansen et al., 2013), and RNA-binding protein (RBP) sponges (Greene et al., 2017; You et al., 2015).

3 CircRNAs are highly stable and detectable in body fluids (Zhang et al., 2018) as well as 84 abundantly expressed (Wang et al., 2014; Zhang et al., 2018) and evolutionarily conserved 85 (Noto, Schmidt \& Matera, 2017) in humans, making them better biomarkers than linear RNAs 
86

87

88

89

90

91

92

93

94

95

96

97

98

100

101

102

103

104

105

106

107

108

109

110

111

112

113

with potential value in clinical diagnosis, therapeutic, and prognostic applications (Wang et al., 2018; Kun-Peng et al., 2018; Xing et al., 2018).

Recent studies have reported the potential of circRNAs as biomarkers (Kun-Peng et al., 2018; Xing et al., 2018; Haque \& Harries, 2017; Zhang et al., 2017) to detect diseases in Drosophila and humans, especially cancer. In this review, we discuss the characteristics and functions of circRNAs. Moreover, we review the current research on circRNAs in CVDs, providing evidence for the significance of circRNAs in CVD diagnosis and clinical treatment.

\section{STUDY DESIGN}

\section{Survey methodology}

Scholarly articles that were reviewed in this paper were searched in journal databases and subject-specific professional websites. The search terms that were used to search the articles included circular RNAs, potential biomarkers, therapeutic, and cardiovascular disease. Inclusion criteria for selected articles required that articles be directly related to the topic on circular RNAs and be peer reviewed.

\section{VALIDITY OF THE FINDINGS}

\section{Biogenesis of circRNAs}

In contrast to linear RNAs, circRNAs lack a 5' cap and 3' polyadenylated tail. They are spliced from pre-mRNA and specially formed with closed covalent bonds (Wilusz \& Sharp, 2013).

There are three different types of circRNAs: exonic, intronic, and exon-intron circRNAs (Li et al., 2015). Recent evidence indicated that exonic circRNAs are formed when a 3' splice donor attaches to the 5' splice acceptor of a single exon. This type of circRNA accounts for more than $80 \%$ of all types of circRNAs. Occasionally, the start of an upstream exon splices and attaches to the other end of a downstream exon, a process referred to as back-splicing process. Thus, a spliced donor joins an acceptor site to form a circular transcript, after which introns are spliced out (Zhang et al., 2016) (Figure 2(a)). However, if the intron is retained during this process, an 
114 exon-intron circRNA is formed (Figure 2(b)). The intronic circRNAs always form from intron

115 lariats, which contain a single unique 2' $-5^{\prime}$ linkage. GU-rich sequences near the $5^{\prime}$ splice site and

116 C-rich sequences near the branch point bind into a circle, and then exonic and intronic sequences

117 are cut out with the remaining introns being brought together to form intronic circRNA

118 (Taborda, Ramírez \& Bernal, 2017) (Figure 2(c)).

119 Emerging studies report another mode of circRNA biogenesis that depends on RBPs such as 120 Quaking (QKI) (Conn et al., 2015) and Muscleblind (MBL) (Ashwal-Fluss et al., 2014), which 121 bridge two flanking introns close together (Figure 2(d)). Another RBP, an adenosine deaminase 122 acting on RNA-1 (ADAR1) (Song et al., 2016), can stop prevent circRNA formation by melting 123 the stem structure (Figure 2(b)). Meanwhile, some studies have revealed that heterogeneous 124 nuclear ribonucleoprotein (hnRNP) and serine-arginine (SR) proteins regulate the expression of 125 circRNAs in Drosophila (Kramer et al., 2015), suggesting that RBPs also play an important role 126 in regulating the levels of circRNAs.

127

128 CircRNAs as miRNA sponges

129 CircRNAs have been reported to function as miRNA sponges by mediating the downregulation 130 or upregulation of miRNA target gene expression (Thomson \& Dinger, 2016). They are found to 131 negatively regulate gene expression by absorption and sequestration of miRNA molecules 132 (Figure 3). miRNAs are a class of small ncRNAs that play an important role in regulating gene 133 expression through the repression of mRNAs. ciRS-7/CDR1as and Sry are representative 134 circRNAs that function as miRNA sponges (Huang et al., 2018; Li et al., 2018). They have 16 135 miR-138 and 74 miR-7 binding sites, respectively. Overexpression or knockdown of ciRS-

$1367 / \mathrm{CDR} 1$ as or Sry results in synchronous increased or decreased expression of the relevant 137 miRNAs, respectively. It is interesting that some circRNAs contain multiple binding sites for a 138 single miRNA. For example, circHIPK3 contains 9 binding sites for growth-suppressive 139 miRNAs (Zheng et al., 2015). On the other hand, some circRNAs, unlike circHIPK3, contain 140 only two binding sites for miR-124, and yet are able to regulate the function/expression of this 141 miRNA. Thus, although multiple binding sites may not be a prerequisite for their regulatory 142 function, circRNAs with multiple binding sites may affect the expression of more miRNA 143 targets. It is still unclear whether a single miRNA binding site is sufficient for the miRNA 144 sponging function of circRNAs. 
146 CircRNAs as RBP sponges

147 RBPs play an important role in post-transcriptional regulatory processes associated with cell 148 differentiation, proliferation, apoptosis, and oxidative stress (Abdelmohsen et al., 2008). Study 149 shows that specific RBPs can influence the lifecycle of an mRNA. Recent research showed that 150 circRNAs could function as 'super-sponges' with a given RBP and thereby regulate the target 151 gene (Dudekula et al., 2016). The hsa_circ_0000020 contains 6 binding sites for human antigen $152 \mathrm{R}(\mathrm{HuR})$ and 10 sites for fragile mental retardation protein (FMRP), while the hsa_circ_0024707 153 contains 85 binding sites for argonaute 2 (AGO2). These circRNAs can store, sort, deliver, and 154 regulate RBPs owing to the high density of binding sites (Hentze \& Preiss, 2012). Another study 155 showed that circPABPN1 can competitively bind to HuR to prevent polyadenylate binding 156 protein 1 (PABPN1) mRNA from binding to HuR in order to influence the translation process 157 (Abdelmohsen et al., 2017). Another recent study suggested that circular antisense non-coding 158 RNA in the INK4 locus (ANRIL) transcripts may regulate the INK4/ARF coding transcripts by 159 competitive splicing (Burd et al., 2010).

\section{CircRNAs as transcriptional regulators}

162 Misregulation of alternative splicing is associated with the aberrant expression of splicing 163 factors. Research shows that circRNAs can act as splicing isoforms and function in regulating 164 alternative splicing. Nuclear exon-intron circRNAs (EIciRNAs) can regulate transcription. The 165 intronic sequence of these specific circRNAs can interact with the U1 component of the spliceosomal machinery and promote the expression of the target genes through recruiting RNA polymerase (Pol) II to the promoter region of genes (Qin, Wei \& Sun, 2018). For example, the circRNA ciankrd52 can act as a positive regulator of Pol II to promote ankyrin repeat domain 52 (ANKRD52) gene transcription (Zhang et al., 2013). CircRNAs can also influence the translation of the cognate linear mRNA and thereby regulate protein expression. For example, circPABPN1 can interact with its cognate mRNA, PABPN1, to reduce the PABPN1 translational efficiency

172 (Abdelmohsen et al., 2017). The circRNA EIciEIF3j could combine with snRNPs (small nuclear 173 ribonucleoproteins) and Pol II to promote its parent gene EIF3J transcription (Elantak et al., 174 2010; ElAntak et al., 2007). Recent reports have shown that ci-mcm5 and ci-sirt7 can enhance 175 the expression of RNA associated with tumorigenesis (Li et al., 2015). In addition, circRNAs can 176 inhibit mRNA maturation. For example, circANRIL can bind to the C-terminal lysine-rich 
177 domain of pescadillo (PES)1, thereby inhibiting pre rRNA processing and impairing ribosome

178 assembly and translation processes (Burd et al., 2010)

179

180

CircRNAs as competitors of linear splicing

181

182

183

184

185

186

187

188

189

190

191

192

193

194

195

196

197

198

199

200

201

202

203

204

205

206

207

A common pre-mRNA may produce many isoforms. The MBL gene contains sequences that form a circRNA transcript having binding sites for MBL itself. Subsequently, in an autoregulatory manner, circMBL influences the selective splicing of the MBL mRNA. Further, MBL can interact with circMBL and its flanking introns to promote exon circularization. In addition, the competition between canonical splicing and circRNA generation is evident from the concomitant decrease in circRNA and increase in linear splicing(Ashwal et al., 2014).

\section{CircRNAs as protein/peptide translators}

Although classified as ncRNA, a recent report showed that circRNAs could be translated into proteins. In fact, the human transcriptome seems to contain many circRNAs with coding potential (Yang et al., 2017). These have been associated in vivo with the translation of polysomes of Drosophila. Circ-ZNF609 was able to translate a GFP protein during myogenesis because it contains an open reading frame at the start codon as the linear transcript (Legnini et al., 2017). However, because these target circRNAs have no free 5' and 3 ' ends, this translation process occurred through rolling circle amplification (RCA) mechanism, driven by internal ribosome entry sites (IRES) and in a 5'-cap-independent manner. The circ-FBXW7 was found to encode functional proteins in human U251 and U373 cell lines (Yang et al., 2018). eIF4G2, YTHDF3, and N6-meythyl adenosine residues, as well as methyltransferase METTL3/14 were found to promote the translation initiation of circRNAs (Yang et al., 2017). Although the protein translation efficiency of target circRNAs is lower in human and murine cells, the hepatitis D virus antigen (HDAg) encoded by circRNAs is-exists after infecting eukaryotic cells. In addition, mRNA modifications of m6A, $\Psi$, and $\mathrm{m} 5 \mathrm{C}$ are important for circRNA translation process, and can alter the efficiency and fidelity of translation (Gilbert, Bell \& Schaening, 2016).

A recent report has shown that circRNAs can also promote protein-protein interactions. For example, CDK2 is a key marker of cell progression from G1 to S phase (Du et al., 2017). CircFoxo3 can interact with CDK2 via p21 to inhibit cell cycle progression. Recently, a circRNA was shown to decrease the nuclear translocation of its co-localized proteins, ID1 and E2F1, and 
208 to also decrease the distribution of HIF1 $\alpha$ (Corcoran \& O'Neill, 2017), indicating that circRNAs 209 can also affect protein localization.

210

circRNAs as potential diagnostic and prognostic biomarkers of cardiovascular disease

212

Linear RNA molecules have been reported as potential biomarkers in several diseases,

213 especially cancer (Wu et al., 2017). However, circRNAs were identified to have more

214 advantages than linear RNAs as biomarkers (Xia et al., 208). It is an ancient, evolutionarily 215 conserved feature in humans and mice, as well as Drosophila. They are more abundant than 216 expected and more stable than linear RNAs due to the covalently closed loop structures, which 217 can resist RNA exonuclease and RNase R activity. In addition, the half-life of stable ncRNAs is 218 about 48 hours, which is much more than that of mRNAs (10 hours) (Ayupe \& Reis, 2017).

219 Moreover, they are located in the cytoplasm, which can be easily acquired and examined. 220 For a long time, biomarkers have been used for early diagnosis and as indicators of the 221 severity of abnormal processes as well as to predict treatment outcomes. An ideal biomarker 222 must be sufficiently variable under normal and diseased conditions for efficient diagnosis. At the 223 same time, it must be easily acquired from blood or bodily fluids. Although many biomarkers 224 have been used in clinical practices, there is still a need for biomarkers with more stability, 225 sensitivity, and specificity.

226 CircRNAs occupy up to $1 \%$ of the total RNA and are differently (via specific isoforms) or 227 specifically expressed in various types of cells (Salzman et al., 2013), suggesting that their 228 expression could be associated with different conditions and consequently could serve as specific biomarkers. Furthermore, circRNAs are relatively abundant in different cells. Reports show that they show higher abundance in low-proliferating cells, such as in the brain, but are relatively lower in number in high-proliferating cells such as in the liver. It is hypothesized that as cells proliferate, circRNAs get divided into the daughter cells, resulting in a total lower abundance of the circRNAs in highly proliferating cells (Bachmayr-Heyda et al., 2015; Nair et al., 2016). This is also evident in highly proliferating tumor cells, which show lower levels of circRNAs than normal tissues (Bachmayr-Heyda et al., 2015).

Memczak et al. (2015) proved the existence of circRNAs in the blood. However, it is more important to know if the amount of circRNA in blood can be easily detected. Predictably, the abundance of circRNAs in blood is higher than that in tissues but lower than that in the brain. 
239 Considering that the low amounts of circRNAs in liver have been suggested as biomarkers

240 (Zhang et al., 2018), we propose that the relatively more abundant blood circRNAs may

241 efficiently serve as biomarkers. Recent research confirmed that circRNAs are also abundant in

242 exosomes, which can be a potential new way to employ circRNAs for disease diagnosis and

243 prognosis (Li et al., 2015). Although exosomes contain some unwanted proteins, DNA, and

244 RNA, these molecules can act as messenger shuttles between cells (Pillay et al., 2017).

245 Therefore, exosomes are potential biomarkers, which have been used for diagnosing multiple

246 diseases as well as assessing responses to drug treatments. Although circRNAs are lower in high-

247 proliferating cells, they are higher in exosomes in such cells than in normal exosomes.

248 Additionally, it is possible that a larger number of exosomes are shed from particular sites in the

249 higher proliferating cells than in normal cells. Together, this suggests that detecting exosomes in 250 such tissues may be more promising to diagnose diseases.

251 Although the total amount of circRNAs is low in body fluids such as blood, saliva, and gastric

252 fluid and other tissues, it is still higher than that of linear RNAs. Multiple reports have proposed

253 that circRNAs as disease biomarkers are superior to the corresponding mRNAs and lncRNAs in

254 terms of abundance, stability, and specificity. The diagnosis and prediction of stage

255 characteristics of a disease are also better with circRNAs than with mRNAs and lncRNAs.

256 Additionally, it is easier and more convenient to detect circRNAs in blood samples, saliva, and

257 gastric juice. Research has identified 422 circRNAs in human cell-free saliva, and these have

258 been implicated in intercellular signaling and inflammatory responses (Bahn et al., 2015).

259 CVD has been reported to be responsible for $31 \%$ of all global deaths. CVDs include coronary 260 artery disease (CAD), aneurysm, MI, and related diseases such as HF and pulmonary arterial 261 hypertension (PAH). Traditional treatment strategies such as controlling risk factors and early 262 treatment after diagnosis result in poor prognosis. Although various biomarkers for CVD have 263 been used for several years, the diagnosis and subsequent treatment often occurs very late as 264 these biomarkers reach significant detectable levels at later disease stages. BNP has been used 265 for the diagnosis of HF, cardiac TnT/I and CK-MB have been utilized for the diagnosis of MI, 266 and D-dimer has been used for the diagnosis of aortic dissection. However, there is still a lack of 267 biomarkers for CAD and other CVDs. In recent years, ncRNAs such as miRNAs and lncRNAs 268 have been suggested as potential biomarkers for CVD. However, it is as yet unclear if circRNAs 
269

270

271

272

273

274

275

276

277

278

279

280

281

282

283

284

285

286

287

288

289

290

291

292

293

294

295

296

297

298

299

could be potential new biomarkers for CVD. Therefore, we discuss below recent discoveries that illustrate the potential of using circRNAs as diagnostic and prognostic CVD biomarkers.

\section{Coronary artery disease and atherosclerosis}

CAD has been a heavy burden on social economy mainly due to its late diagnosis and severe complications. CAD and atherosclerosis are linked to endothelial injury and imbalance of lipid metabolism. The important roles of miRNAs in atherosclerosis, such as regulation of vascular smooth muscle cell function, lipid homeostasis, and cytokine responsiveness, have been described thoroughly (Feinberg \& Moore, 2016; Markus et al., 2016). MI and HF are the main results of CAD and lead to severe mortality. CircANRIL can regulate cell functions related to atherosclerosis and plays an atheroprotective role. The antisense ANRIL and the INK4/ARF genes are found on chromosome 9p21.3 (Wellcome Trust Case Control Consortium, 2007). Patients with high circANRIL expression develop less severe CAD. This circRNA can impair the process of exonuclease-mediated pre-rRNA and ribosome biogenesis by binding to PES1. Consequently, this induces apoptosis and inhibition of proliferation of vascular smooth muscle cells and macrophages, which can promote anti-atherogenic cell stability and prevent degradation (Burd et al., 2010). It also can affect atherosclerosis by regulating INK4/ARF expression. Thus, stable circANRIL may act as a potential biomarker and therapeutic target for atherosclerosis (Sankar et al., 2017; Holdt et al., 2016).

Pan et al. (2017) used circRNA microarray to detect differentially expressed circRNAs between three samples from CAD patients and three control plasma samples. They found hsa_circ_0006323, hsa_circ_0032970, hsa_circ_0051172, hsa_circ_0054537, hsa_circ_0057576, hsa_circ_0068942, hsa_circ_0082824, hsa_circ_0083357, and hsa_circ_0089378 to be differentially expressed with fold-change $\geq 1.5$ and $P<0.05$. These nine circRNAs act as an hsamiR-130a-3p sponge to influence its target mRNA, transient receptor potential cation channel subfamily M member 3 (TRPM3). TRPM3 regulates contractility and proliferation of vascular smooth muscle cells in coordination with cholesterol, which plays an important role in CAD. However, these results need to be verified using a larger sample size of CAD patients and normal individuals. Zhao et al. (2017) used the same method to show differences between the peripheral blood of 12 patients with CAD and 12 normal individuals and found that hsa_circ_0124644 and hsa_circ_0082081 were significantly associated with CAD. In addition, they found that 
300 hsa_circ_0124644 in blood could act as a potential diagnostic biomarker for CAD with a

301 specificity and sensitivity of 0.626 and 0.861 , respectively.

302

303

\section{Myocardial fibrosis}

304

305

Myocardial fibrosis is a disease of myocardial stiffness, which can reduce myocardial

306

shortening to induce diastole difficulty. Endothelial-to-mesenchymal transition (EndMT) is the

307

process of inducing normal endothelial cells into mesenchymal-like cells. Tissue fibrosis is

308 associated with fibroblast-specific protein-1 (FSP-1) expression and collagen deposition. The profibrotic factor, transforming growth factor $\beta$ (TGF- $\beta$ ), is able to drive EndMT progression. It has been shown that TGF- $\beta 1$-mediated induction of $\alpha$-SMA expression and concomitant loss of VE-cadherin expression in aortic endothelial cells results in EndMT. Three EndMT-related circRNAs, chr5:90817794|90827570, chr8:71336875|71337745, and chr6:22033342|22038870, were found to be significantly upregulated in TGF- $\beta 1$-treated rat coronary artery endothelial cells (CAEC). These three circRNAs may be potential biomarkers of EndMT-induced myocardial fibrosis (Huang et al., 2018). with fold change $>3$ and $P<0.05$ between diabetic $\mathrm{db} / \mathrm{db}$ mice and $\mathrm{db} / \mathrm{m}$ control mice. They selected one of the markedly increased circRNAs (circRNA_010567) for further bioinformatics analysis and found it to contain binding sites for miR-141, which is predicted to regulate TGF- $\beta 1$ function. Further, results suggested that circRNA_010567 could directly target miR-141 and regulate TGF- $\beta 1$ expression, which can mediate resection-associated fibrosis. These results imply that circRNA_010567 may play a key role in myocardial fibrosis, and thus provide a novel insight into cardiopathy pathogenesis and act as a potential diagnostic biomarker.

Tang et al. (2017) reported a new target, circRNA_000203, which was significantly upregulated in diabetic myocardium. This circRNA can act as a sponge for miR-26b-5p to restrain its downstream targets, Col1a2 and CTGF, thus promoting pro-fibrosis effects. Although circRNA_000203 may play important roles in myocardial fibrosis, its efficacy as a biomarker needs further investigation.

330 Cardiomyopathy is a disease with primary abnormalities in the structure and function of the heart. Dilated cardiomyopathy is a morphological subtype of this disease. Siede et al. (2017) 
332 reported increased expression of three circRNAs SLC8A1, CHD7, and ATXN10 relative to their

333 host gene expression and decreased circDNA6JC expression in patients with dilated

334 cardiomyopathy. Xie et al. (2016) found that Foxo3 is the protective agent in Ganoderma spore

335 oil against cardiomyopathy. In addition, circAmotl1 was associated with cardiac dysfunction,

336 wherein it could promote cardiac repair through binding AKT1 and PDK1 to accelerate the

337 function of cardioprotective nuclear translocation of pAKT. This may provide a way to detect

338 cardiac dysfunction before HF. circAmotll was also shown to be important for ameliorating the

339 negative effects of Dox on the heart, such as resistant fibrosis, apoptosis, and hypertrophy. Thus,

340 circAmotll could have therapeutic potential in the treatment of cardiomyopathy (Zeng et al.,

341 2017). Khan et al. (2016) reported that RBM20-dependent TTN circRNAs play important roles

342 in dilated cardiomyopathy. However, there are only few studies on the role of circRNAs in

343 cardiomyopathy.

344

345

346

347

\section{Heart Failure}

HF is a disease that is often a secondary effect caused by another disease. It is also a final outcome of some CVDs such as CAD, vascular disease, and MI. Therefore, it is challenging to find an ideal biomarker for HF. HF leads to a decrease in systolic and/or diastolic function (Mentz \& O'Connor, 2016). Traditional standard biochemical biomarkers such as BNP have been used to distinguish HF from other conditions (O'Donoghue et al., 2005). However, studies have shown that ncRNAs are more sensitive than traditional biomarkers such as BNP or cardiac troponins. Up till now, more than 50 miRNAs and 3 lncRNAs have been described as biomarkers of $\mathrm{HF}$.

Salgado-Somoza et al. (2017) reported use of the circRNA, myocardial infarction-associated circular RNA (MICRA), to predict the risk in MI patients. Using blood samples from 472 acute MI patients, they found that the expression levels of MICRA were lower in patients with ejection fraction $(\mathrm{EF}) \leq 40 \%$ than in patients with $\mathrm{EF}>41 \%$. Patients with lower levels of MICRA were also at high risk of decreased EF (0.78 [0.64-0.95]). They further identified that MICRA was present in $86 \%$ of the samples, and bootstrap internal validation indicated that it could act as an optimal HF predictive biomarker, similar to the traditional markers (Nt-proBNP, creatine phosphokinase, CPK). Wang et al. (2016) reported that a special heart-related circRNA (HRCR) may play an important role in HF by acting as an miR-223 sponge to increase the expression of ARC (apoptosis repressor with CARD domain); however, this requires further investigation. 
364

365

366

367

368

369

370

371

372

373

374

375

376

377

378

379

380

381

382

383

384

385

386

387

388

389

390

391

392

393

394

395

\section{Myocardial injury}

Myocardial injury and apoptosis are associated with HF and MI as well as reperfusion injury. Increase in apoptosis augments MI and HF, whereas reduced apoptosis protects the heart. Wang et al. (2017) found that the circRNA, MFACR, could increase mitochondrial fission and apoptosis as well as cardiomyocyte cell death through downregulation of the ceRNA(competing endogenous RNAs), miR-652-3p, and promotion of MTP18 translation. In this study, MFACR was shown to act as a sponge to inhibit the activity of miR-652-3p, which directly targets

MTP18 to inhibit mitochondrial fission and apoptosis. Thus, MFACR may act as a biomarker to predict apoptosis in the heart and may serve as a potential therapeutic target for treatment.

\section{Myocardial infarction}

MI is a catastrophic condition caused by ischemic heart disease and can lead to heart tissue damage and mortality. Although revascularization can be used as a valuable method to stabilize the crisis, selective biomarkers are required to assess the risk and therapeutic response after the infarction (Hachey et al., 2017). Acute MI poses sizeable morbidity and mortality risks. HsamiR-122-5p levels that are different in the plasma of CVD patients were proposed as an early prognostic biomarker of acute MI (Cortez-Dias et al., 2016).

Through circRNA microarray analysis in mouse hearts with autophagy, Zhou et al. (2018) found that mmu_circRNA_006636 (ACR) was significantly decreased after I/R injury. ACR was found to regulate Pink1 expression through inhibition of DNA methylation of Pink1 by binding to DNMT3B. Further, after verifying the functional role of Pink1 in autophagy, they identified the downstream target of Pink1 as FAM65B. Thus, ACR can repress autophagy and MI by targeting Pink1-mediated phosphorylation of FAM65B, and could act as a potential therapeutic target and biomarker for ischemia/reperfusion and MI.

Geng et al. (2016) found that circRNA CDR1AS could act as an miR-7a sponge to regulate the expression of its target gene (PARP and SP1) and interfere with its protective role in MI injury. In addition, apoptosis-related circRNA MFACR could regulate miR-652-3p to promote the progress of MTP18, which is relevant for MI (Wang et al., 2017). Vausort et al. (2016) reported that MICRA was highly expressed in peripheral blood of MI patients. However, its potential value act as a biomarker for MI remains to be further investigated. Deng et al. (2016) found that circRNA_081881 was differentially expressed in plasma samples of acute MI 
396 patients. This report shows that the downregulated circRNA_081881 can sequester miR-548

397 through competitive binding sites to reduce PPAR $\gamma$ (a heart-protective factor) expression, which 398 is reduced in the plasma of patients with acute MI.

399

400

\section{Hypertension}

401

402

Hypertension has become a major risk factor for the development of CVD and is highly

403

prevalent across the world, even in young people. It has been a major contributor to morbidity

404 and mortality and is considered as a socioeconomic burden. The diagnosis and treatment are influenced by many factors and there is a lack of useful biomolecules for clinical prediction and 405 diagnosis.

Bao et al. (2018) detected differences in the expression of some circRNAs between five pairs 407 of newly diagnosed essential hypertension (EH) and non-EH whole blood samples. They found 408 that hsa_circ_0037911 levels in the EH group were significantly higher than those in the control group $(\mathrm{t}=2.834, P=0.005)$. In addition, they proved that the target hsa_circ_0037911 was an effective predictor of EH by using the ROC (receiver operating characteristic) curve to investigate its diagnostic value (area under curve, $\mathrm{AUC}=0.627 ; P=0.002$ ). Their study also showed that there was a direct correlation between hsa_circ_0037911 and serum creatinine, indicating that this RNA plays an important role in the pathogenesis of essential hypertension.

414 Another study reported that 46 circRNAs were significantly upregulated and 13 downregulated 415 (FC $>2.0$ and $P<0.05$ ) between human hypertensive plasma and normal plasma. hsa-circ0005870 was verified to be significantly downregulated in hypertensive patients. Bioinformatics analysis also indicated that hsa-circ-0005870 may represent a novel biomarker for the diagnosis of hypertension (Wu Jin \& Cai, 2017).

419

420

\section{Aneurysm disease}

421 Aneurysm disease especially aortic dissection is a life-threatening condition with a lethality rate 422 of $1-2 \%$ per hour after onset of symptoms in untreated patients. The treatment is complex and 423 the prognosis is poor, especially Stanford A aortic dissection. However, there is no available 424 biomarker that can reveal this disease before acute onset. Therefore, predictive diagnosis and treatment is vital to improve the survival rate and to prevent severe complications. 
426

427

428

429

430

431

432

433

434

435

436

437

438

439

440

441

442

443

444

445

446

447

448

449

450

451

452

453

454

455

456

457

Zou et al. (2017) reported 106 downregulated and 156 upregulated circRNAs between 3 normal patients and 3 thoracic aortic dissection (TAD) patients. They found hsa_circRNA_101238 to be upregulated with a fold-change $\geq 1.5, P<0.05$, and co-expression network revealed that only this target circRNA interacted with the three altered miRNAs (hsamiR-320a, hsa-miR-320b, and hsa-miR-320c). The circRNA-miRNA-mRNA network predicted miRNA targets of hsa_circRNA_101238 were hsa-miR-320b, hsa-miR-320a, hsa-miR-138-5p, hsa-miR-593-5p, and hsa-miR-320c. Through target gene prediction and luciferase assays, they found that hsa_circRNA_101238 acted as an miR-320a sponge through inhibiting the expression of hsa-miR-320a to increase MMP9 expression, which may be involved in the pathogenesis of TAD. Thus, hsa_circRNA_101238 may be a potential biomarker for TAD. However, the function and mechanism of circRNAs in TAD need further experimental evidence.

\section{Pathological hypertrophy}

Cardiomyopathies are myocardial diseases with morphological and functional abnormalities and can be classified as primary or intrinsic cardiomyopathies. Pathological diagnosis of cardiomyopathies requires invasive and potentially dangerous tests. Therefore, many patients with cardiomyopathies usually opt for biochemical tests that can help diagnosis (Coats et al., 2015).

Meng et al. (2019) reported differentially expressed circRNAs in cardiac hypertrophy cells cultured in the presence of high and normal levels of D-glucose. Five circRNAs, namely ciRNA261, ciRNA26, circRNA1191, circRNA4251, and circRNA6913, were found to be significantly differentially expressed $(P<0.05$ and fold change $>2$ or $<0.5)$ and had more than 60 target miRNAs, which implied that these circRNAs may play important roles in cardiac hypertrophy and potentially serve as biomarkers.

Wang et al. (2017) reported that heart-related circRNA (HRCR) could act as an endogenous miR-223 sponge and inhibit the activity of miR-223. In addition, they also reported that miR-223 is functionally related to hypertrophy through ARC (apoptosis repressor with caspase recruitment domain ). Thus, HRCR inhibits miR-223 activity, resulting in increased expression of its downstream target ARC and inhibition of cardiac hypertrophy and heart failure in mice. Thus, HRCR may be a useful biomarker for diagnosis and prognosis of cardiac hypertrophy and HF. 
458 Cardiac senescence

459 Cardiac senescence accompanies ageing and may decrease heart function. Chen et al. (2018)

460 reported that aging significantly affects the cardiac muscle. By using high throughput RNA

461 sequencing, they found 21 up-regulated circRNAs and 1 down-regulated gene in cardiac muscle

462 during aging. Within the network, circRNA005698 was found to be associated with 7 miRNAs.

463 Further, they found that circRNAs including circRNA005698 might play a key role in regulating

464 pro-coagulation process during aging. Thus, circRNA005698 can act as a biomarker for cardiac 465 senescence.

466 Du et al. (2017) also found that circFoxo3 is relevant for cell senescence in doxorubicin467 induced mouse cardiomyopathy. This circRNA could interact with senescence-related proteins 468 (ID1 and E2F1) and stress-related proteins (HIF1 $\alpha$ and FAK) in the cytoplasm, leading to the 469 inhibition of the anti-stress and anti-senescent roles of these proteins and consequently 470 promoting cardiac senescence. circFoxo3 is not only found in aged heart of mice but also in 471 humans.

472 4. General comments

\section{Conclusions}

474 In summary, circRNAs are a class of ncRNAs that mainly regulate gene expression.

475 Increasing evidence indicates that circRNAs are abundantly found in saliva, exosomes, and 476 clinical standard blood samples, which make them promising biomarkers for disease diagnosis 477 and outcome prediction. They are more stable and sensitive as well as specific than standard 478 biomarkers. In CVD, secreted lncRNAs have been described as biomarkers for several 479 conditions including MI, cardiac failure, and atrial fibrillation. Hopefully, in the future, the use 480 of circRNAs as biomarkers will become routine in clinical practice.

481

482 Limitations

483 To be able to use circRNAs as routine biomarkers, there are some limitations that need to be 484 addressed. First, several methodological factors including sample collection and processing, as 485 well as assay performance and ncRNA quantification can influence the quality of the resulting 486 data and need to be improved. Second, analysis of case studies can be limited by the sample size 487 due to lack of statistical power. In addition, sex, age, and the diversity of the cardiovascular risk 488 factors may introduce bias in limited samples. Therefore, large cohorts and multi-center studies 
489 are necessary to interpret data and make conclusions. Third, patients with CVD are likely to take 490 anticoagulant drugs such as aspirin and clopidogrel before blood collection, which can alter 491 circulating ncRNA concentrations and thereby their quantification. Fourth, primer design and 492 normalization in quantitative real-time polymerase chain reaction can influence the outcome of 493 biomarker studies. Therefore, it is necessary to standardize such procedures to eliminate 494 technical and analytical variability.

495

496

497

498

499

500

501

\section{Acknowledgements}

503 None

504

505

506

507

\section{References}

509 Abdelmohsen K, Kuwano Y, Kim HH, Gorospe M. 2008. Posttranscriptional gene regulation by 510 RNA-binding proteins during oxidative stress: implications for cellular senescence. Biological 511 Chemistry 389:243-255.

512 Abdelmohsen K, Panda AC, Munk R, Grammatikakis I, Dudekula DB, De S, Kim J, Noh JH, 513 Kim KM, Martindale JL, Gorospe M. 2017. Identification of HuR target circular RNAs 514 uncovers suppression of PABPN1 translation by circPABPN1. RNA Biology 14:361-369. 
515 Ashwal-Fluss R, Meyer M, Pamudurti NR, Ivanov A, Bartok O, Hanan M, Evantal N, Memczak

516

517

518

519

520

521

522

523

524

525

526

527

528

529

530

531

532

533

534

535

536

537

538

539

540

541

542

543

544

545

546

547

548

549

550

551

552

553

554

S, Rajewsky N, Kadener S. 2014. circRNA biogenesis competes with pre-mRNA splicing. Molecular Cell 56:55-66.

Ayupe AC, Reis EM. 2017. Evaluating the stability of mRNAs and noncoding RNAs. Methods in Molecular Biology 1468: 139-153 DOI: 10.1007/978-1-4939-4035-6_11.

Bachmayr-Heyda A, Reiner AT, Auer K, Sukhbaatar N, Aust S, Bachleitner-Hofmann T, Mesteri I, Grunt TW, Zeillinger R, Pils D. 2015. Correlation of circular RNA abundance with proliferation-exemplified with colorectal and ovarian cancer, idiopathic lung fibrosis, and normal human tissues. Scientific Report 5:8057. doi:10.1038/srep08057.

Bahn JH, Zhang Q, Li F, Chan TM, Lin X, Kim Y, Wong DT, Xiao X. 2015. The landscape of microRNA, Piwi-interacting RNA, and circular RNA in human saliva. Clinical Chemistry 61:221-230 DOI: 10.1373/clinchem.2014.230433

Bao X, Zheng S, Mao S, Gu T, Liu S, Sun J. 2018. A potential risk factor of essential hypertension in case-control study: Circular RNA hsa_circ_0037911. Biochemical and Biophysical Research Communications 498:789-794.

Barwari T, Joshi A, Mayr M. 2016. MicroRNAs in cardiovascular disease. Journal of the American College of Cardiology 68: 2577-2584.

Burd CE, Jeck WR, Liu Y, Sanoff HK, Wang Z, Sharpless NE. 2010. Expression of linear and novel circular forms of an INK4/ARF-associated non-coding RNA correlates with atherosclerosis risk. PLoS Genetics 6:e1001233 (2010).

Calzetta L, Orlandi A, Page C, Rogliani P, Rinaldi B, Rosano G, Cazzola M, Matera MG. 2016. Brain natriuretic peptide: much more than a biomarker. International Journal of Cardiology 221:1031-1038 (2016) DOI: 10.1016/j.ijcard.2016.07.109.

Chen J, Zou Q, Lv D, Wei Y, Raza MA, Chen Y, Li P, Xi X, Xu H, Wen A, Zhu L. 2018. Comprehensive transcriptional landscape of porcine cardiac and skeletal muscles reveals differences of aging. Oncotarget 9:1524-15414.

Coats CJ, Heywood WE, Mills K, Elliott PM. 2015. Current applications of biomarkers in cardiomyopathies. Expert Review of Cardiovascular Therapy 13:825-837.

Conn SJ, Pillman KA, Toubia J, Conn VM, Salmanidis M, Phillips CA, Roslan S, Schreiber AW, Gregory PA, Goodall GJ. 2015. The RNA binding protein quaking regulates formation of cir-cRNAs. Cell 160:1125-1134.

Corcoran SE, O'Neill LA. 2016. HIF1 $\alpha$ and metabolic reprogramming in inflammation. The Journal of Clinical Investigation 126:3699-3707 DOI: 10.1172/JCI84431.

Cortez-Dias N, Costa MC, Carrilho-Ferreira P, Silva D, Jorge C, Calisto C, Pessoa T, Martins SR, de Sousa JC, da Silva PC, Fiúza M. 2016. Circulating miR-122-5p/miR-133b ratio is a specific early prognostic biomarker in acute myocardial infarction. Circulatory Journal 80:2183-2191.

Deng YY, Zhang W, She J, Zhang L, Chen T, Zhou J, Yuan Z. 2016. GW27-e1167 circular RNA related to PPAR [gamma] function as ceRNA of microRNA in human acute myocardial infarction. Journal of the American College of Cardiology 68: C51-C52.

Peer) reviewing PDF | (2019:01:34077:2:0:NEW 16 Mar 2019) 
555 Djebali S, Davis CA, Merkel A, Dobin A, Lassmann T, Mortazavi A, Tanzer A, Lagarde J, Lin

556

557

558

559

560

561

562

563

564

565

566

567

568

569

570

571

572

573

574

575

576

577

578

579

580

581

582

583

584

585

586

587

588

589

590

591

592

593

594

W, Schlesinger F, Xue C. 2012. Landscape of transcription in human cells. Nature 489:101108.

Du WW, Yang W, Chen Y, Wu ZK, Foster FS, Yang Z, Li X, Yang BB. 2017. Foxo3 circular

RNA promotes cardiac senescence by modulating multiple factors associated with stress and senescence responses. European Heart Journal 38:1402-1412.

Dudekula DB, Panda AC, Grammatikakis I, De S, Abdelmohsen K, Gorospe M. 2016. CircInteractome: a web tool for exploring circular RNAs and their interacting proteins and microRNAs. RNA Biology 13:34-42.

Ebbesen KK, Hansen TB, Kjems J. 2017. Insights into circular RNA biology. RNA Biology 14:1035-1045 DOI: 10.1080/15476286.2016.1271524.

ElAntak L, Tzakos AG, Locker N, Lukavsky PJ. 2007. Structure of eIF3b RNA recognition motif and its interaction with eIF3j: structural insights into the recruitment of eIF3b to the 40 S ribosomal subunit. Journal of Biological Chemistry 282:8165-8174.

ElAntak L, Wagner S, Herrmannová A, Karásková M, Rutkai E, Lukavsky PJ, Valášek L. 2010. The indispensable N-terminal half of eIF3j/HCR1 cooperates with its structurally conserved binding partner eIF3b/PRT1-RRM and with eIF1A in stringent AUG selection. Journal of Molecular Biology 396:1097-1116 DOI: 10.1016/j.jmb.2009.12.047.

Enroth S, Johansson Å, Enroth SB, Gyllensten U. 2014. Strong effects of genetic and lifestyle factors on biomarker variation and use of personalized cutoffs. Nature Communications 5:4684 DOI: $10.1038 /$ ncomms5684.

Fan X, Weng X, Zhao Y, Chen W, Gan T, Xu D. 2017. Circular RNAs in cardiovascular disease: an overview. BioMed Research International 2017:5135781 DOI: 10.1155/2017/5135781.

Feinberg MW, Moore KJ. 2016. MicroRNA regulation of atherosclerosis. Circulatory Research 118:703-720.

Frankish A, Diekhans M, Ferreira AM, Johnson R, Jungreis I, Loveland J, Mudge JM, Sisu C, Wright J, Armstrong J, Barnes I. 2018. GENCODE reference annotation for the human and mouse genomes. Nucleic Acids Research 47(Database issue): D766-D773.

Gao Y, Wang J, Zhao F. 2015. CIRI: an efficient and unbiased algorithm for de novo circular RNA identification. Genome Biology 16:4 DOI: 10.1186/s13059-014-0571-3

Geng HH, Li R, Su YM, Xiao J, Pan M, Cai XX, Ji XP. 2016. The circular RNA Cdrlas promotes myocardial infarction by mediating the regulation of miR-7a on its target genes expression. PLoS One 11:e0151753.

Gilbert WV, Bell TA, Schaening C. 2016. Messenger RNA. Modifications: form, distribution, and function. Science 352: 1408-1412.

Greco S, Gorospe M, Martelli F. 2015. Noncoding RNA in age-related cardiovascular diseases. Journal of Molecular and Cellular Cardiology 83:142-155.

Greene J, Baird AM, Brady L, Lim M, Gray SG, McDermott R, Finn SP. 2017. Circular RNAs: biogenesis, function and role in human diseases. Frontiers in Molecular Biosciences 4:38 DOI: 10.3389/fmolb.2017.00038.

Peer) reviewing PDF | (2019:01:34077:2:0:NEW 16 Mar 2019) 
595 Hachey BJ, Kontos MC, Newby LK, Christenson RH, Peacock WF, Brewer KC, McCord J.

596

597

598

599

600

601

602

603

604

605

606

607

608

609

610

611

612

613

614

615

616

617

618

619

620

621

622

623

624

625

626

627

628

629

630

631

632

633

2017. Trends in use of biomarker protocols for the evaluation of possible myocardial infarction. Journal of the American Heart Association 6: 005852.

Hansen TB, Jensen TI, Clausen BH, Bramsen JB, Finsen B, Damgaard CK, Kjems J. 2013. Natural RNA circles function as efficient mi-croRNA sponges. Nature 495:384-388.

Haque S, Harries LW. 2017. Circular RNAs (circRNAs) in health and disease. Genes (Basel) 8:353 DOI: 10.3390/genes8120353.

Heil B, Tang WH. 2015. Biomarkers: their potential in the diagnosis and treatment of heart failure. Cleveland Clinic Journal of Medicine 82: S28-S35 DOI: 10.3949/ccjm.82.s2.05. Hentze MW, Preiss T. 2012. Circular RNAs: splicing's enigma variations. EMBO Journal 32:923-925.

Holdt LM, Stahringer A, Sass K, Pichler G, Kulak NA, Wilfert W, Kohlmaier A, Herbst A, Northoff BH, Nicolaou A, Gäbel G. 2016. Circular non-coding RNA ANRIL modulates ribosomal RNA maturation and atherosclerosis in humans. Nature Communications 7:12429. Huang H, Wei L, Qin T, Yang N, Li Z, Xu Z. 2019. Circular RNA ciRS-7 triggers the migration and invasion of esophageal squamous cell carcinoma via miR-7/KLF4 and NF- $\mathrm{kB}$ signals. Cancer Biology \& Therapy 20:73-80 DOI: 10.1080/15384047.2018.1507254.

Huang X, Chen Y, Xiao J, Huang Z, He L, Xu D, Peng J. 2018. Identification of differentially expressed circular RNAs during TGF- $\beta 1$-induced endothelial-to-mesenchymal transition in rat coronary artery endothelial cells. Anatolian Journal of Cardiology 19:192-197.

Jeck WR, Sorrentino JA, Wang K, Slevin MK, Burd CE, Liu J, Marzluff WF, Sharpless NE. 2013. Circular RNAs are abundant, conserved, and associated with ALU repeats. RNA 19:141-157 DOI: 10.1261/rna.035667.112

Khan MA, Reckman YJ, Aufiero S, van den Hoogenhof MM, van der Made I, Beqqali A, Koolbergen DR, Rasmussen TB, Van Der Velden J, Creemers EE, Pinto YM. 2016. RBM20 regulates circular RNA production from the titin gene. Circulatory Research 119:996-1003.

Kim MC, Ahn Y, Cho KH, Lee MG, Ko JS, Park KH, Sim DS, Yoon NS, Yoon HJ, Kim KH, Hong YJ. 2011. Early statin therapy within 48 hours decreased one-year major adverse cardiac events in patients with acute myocardial infarction. International Heart Journal 52:16.

Kondkar AA, Abu-Amero KK. 2015. Utility of circulating microRNAs as clinical biomarkers for cardiovascular diseases. BioMed Research International 2015: 821823 DOI: $10.1155 / 2015 / 821823$.

Kramer MC, Liang D, Tatomer DC, Gold B, March ZM, Cherry S, Wilusz JE. 2015. Combinatorial control of Drosophila circular RNA expression by intronic repeats, hnRNPs, and SR proteins. Genes \& Development 29:2168-2182.

Kun-Peng Z, Chun-Lin Z, Jian-Ping H, Lei Z. 2018. A novel circulating hsa_circ_0081001 act as a potential biomarker for diagnosis and prognosis of osteosarcoma. International Journal of Biological Sciences 14:1513-1520 DOI: 10.7150/ijbs.27523. eCollection 2018.

Peer) reviewing PDF | (2019:01:34077:2:0:NEW 16 Mar 2019) 
634 Legnini I, Di Timoteo G, Rossi F, Morlando M, Briganti F, Sthandier O, Fatica A, Santini T,

635

636

637

638

639

640

641

642

643

644

645

646

647

648

649

650

651

652

653

654

655

656

657

658

659

660

661

662

663

664

665

666

667

668

669

670

671

672 Andronache A, Wade M, Laneve P. 2017. Circ-ZNF609 is a circular RNA that can be translated and functions in myogenesis. Molecular Cell 66:22.

Li L, Wang L, Li H, Han X, Chen S, Yang B, Hu Z, Zhu H, Cai C, Chen J, Li X. 2018. Characterization of LncRNA expression profile and identification of novel LncRNA biomarkers to diagnose coronary artery disease. Atherosclerosis 275:359-367 DOI: 10.1016/j.atherosclerosis.2018.06.866.

Li M, Wang J, Liu D, Huang H. 2018. High-throughput sequencing reveals differentially expressed lncRNAs and circRNAs, and their associated functional network, in human hypertrophic scars. Molecular Medicine Reports 18:5669-5682 DOI: 10.3892/mmr.2018.9557.

Li Y, Zheng Q, Bao C, Li S, Guo W, Zhao J, Chen D, Gu J, He X, Huang S. 2015. Circular RNA is enriched and stable in exosomes: a promising biomarker for cancer diagnosis. Cell Research 25: 981-984.

Li Z, Huang C, Bao C, Chen L, Lin M, Wang X, Zhong G, Yu B, Hu W, Dai L, Zhu P. 2015. Exon-intron circular RNAs regulate transcription in the nucleus. Nature structural \& Molecular Biology 22:256-264 DOI: 10.1038/nsmb.2959

Markus B, Grote K, Worsch M, Parviz B, Boening A, Schieffer B, Parahuleva MS. 2016. Differential expression of MicroRNAs in endarterectomy specimens taken from patients with asymptomatic and symptomatic carotid plaques. PLoS One 11:e0161632.

Memczak S, Jens M, Elefsinioti A, Torti F, Krueger J, Rybak A, Maier L, Mackowiak SD, Gregersen LH, Munschauer M, Loewer A. 2013. Circular RNAs are a large class of animal RNAs with regulatory potency. Nature 495:333-338.

Memczak S, Papavasileiou P, Peters O, Rajewsky N. 2015 Identification and characterization of circular RNAs as a new6 class of putative biomarkers in human blood. PLoS One 10:e0141214 (2015) DOI:10.1371/journal.pone.0141214

Meng Z, Chen C, Cao H, Wang J, Shen E. 2019. Whole transcriptome sequencing reveals biologically significant RNA markers and related regulating biological pathways in cardiomyocyte hypertrophy induced by high glucose. Journal of Cellular Biochemistry 120:1018-1027.

Mentz RJ, O’Connor CM. 2016. Pathophysiology and clinical evaluation of acute heart failure. Nature Reviews Cardiology 13:28-35.

Nair AA, Niu N, Tang X, Thompson KJ, Wang L, Kocher JP, Subramanian S, Kalari KR. 2016. Circular RNAs and their associations with breast cancer subtypes. Oncotarget 7:8096780979 DOI: 10.18632/oncotarget.13134.

Ng WL, Marinov GK, Liau ES, Lam YL, Lim YY, Ea CK. 2016. Inducible RasGEF1B circular RNA is a positive regulator of ICAM-1 in the TLR4/LPS pathway. RNA Biology 13:861-871. Noto JJ, Schmidt CA, Matera AG. 2017. Engineering and expressing circular RNAs via tRNA splicing. RNA Biology 14:978-984 DOI: 10.1080/15476286.2017.1317911.

Peer) reviewing PDF | (2019:01:34077:2:0:NEW 16 Mar 2019) 
673 O'Donoghue M, Chen A, Baggish AL, Anwaruddin S, Krauser DG, Tung R, Januzzi Jr JL.

674

675

676

677

678

679

680

681

682

683

684

685

686

687

688

689

690

691

692

693

694

695

696

697

698

699

700

701

702

703

704

705

706

707

708

709

710

711

(2005). The effects of ejection fraction on N-terminal ProBNP and BNP levels in patients with acute CHF: analysis from the ProBNP Investigation of Dyspnea in the Emergency Department (PRIDE) study. Journal of Cardiac Failure 11:S9-14.

Pan RY, Liu P, Zhou HT, Sun WX, Song J, Shu J, Cui GJ, Yang ZJ, Jia EZ. 2017. Circular RNAs promote TRPM3 expression by inhibiting hsa-miR-130a-3p in coronary artery disease patients. Oncotarget 36:60280-60290.

Pillay P, Moodley K, Moodley J, Mackraj I. 2017. Placentaderived exosomes: potential biomarkers of preeclampsia. International Journal of Nanomedicine 12:8009-8023 DOI: 10.2147/IJN.S142732.

Qin M, Wei G, Sun X. 2018. Circ-UBR5: An exonic circular RNA and novel small nuclear RNA involved in RNA splicing. Biochemical and Biophysical Research Communications 503:1027-1034 DOI: 10.1016/j.bbrc.2018.06.112.

Salgado-Somoza A, Zhang L, Vausort M, Devaux Y. 2017. The circular RNAMICRA for risk stratification after myocardial infarction. International Journal of Cardiology, Heart \& Vasculature 17:33-36.

Salzman J, Chen RE, Olsen MN, Wang PL, Brown PO. 2013. Cell-type specific features of circular RNA expression. PLoS Genetics 9:e1003777 DOI: 10. 1371/journal.pgen.1003777.

Salzman J, Gawad C, Wang P L, Lacayo N, Brown PO. 2012. Circular RNAs are the predominant transcript isoform from hundreds of human genes in diverse cell types. PLoS One 7:e30733 DOI: 10.1371/journal.pone.0030733

Sanger HL, Klotz G, Riesner D, Gross HJ, Kleinschmidt AK. 1976. Viroids are single-stranded covalently closed circular RNA molecules existing as highly base-paired rod-like structures.

Proceedings of the National Academy of Sciences 73: 3852-3856 DOI: 10.1073/pnas.73.11.3852.

Sarkar D, Oghabian A, Bodiyabadu PK, Joseph WR, Leung EY, Finlay GJ, Baguley BC, Askarian-Amiri ME. 2017. Multiple isoforms of ANRIL in melanoma cells: structural complexity suggests variations in processing. International Journal of Molecular Sciences 18: E1378 DOI: 10.3390/ijms 18071378.

Shi Q, Yang X. 2016. Circulating microRNA and long noncoding RNA as biomarkers of cardiovascular diseases. Journal of Cellular Physiology 231:751-755 DOI: $10.1002 /$ jcp. 25174.

Siede D, Rapti K, Gorska AA, Katus HA, Altmüller J, Boeckel JN, Meder B, Maack C, Völkers M, Müller OJ, Backs J. 2017. Identification of circular RNAs with host gene-independent expression in human model systems for cardiac differentiation and disease. Journal of Molecular and Cellular Cardiology 109:48-56 DOI: 10.1016/j.yjmcc.2017.06.015.

Song C, Sakurai M, Shiromoto Y, Nishikura, K. 2016. Functions of the RNA editing enzyme ADAR1 and their relevance to human diseases. Genes (Basel) 7: E129 DOI: 10.3390/genes 7120129 . 
712 Suzuki H, Tsukahara T. 2014. A view of pre-mRNA splicing from RNase R resistant RNAs.

713 International Journal of Molecular Sciences 15:9331-9342.

714 Taborda MI, Ramírez S, Bernal G. 2017. Circular RNAs in colorectal cancer: Possible roles in

715 regulation of cancer cells. World Journal of Gastrointestinal Oncology 9:62-69 DOI:

716 10.4251/wjgo.v9.i2.62.

717 Taft RJ, Pang KC, Mercer TR, Dinger M, Mattick JS. 2010. Non-coding RNAs: regulators of 718 disease. The Journal of Pathology 220:126-39.

719 Tang CM, Zhang M, Huang L, Hu ZQ, Zhu JN, Xiao Z, Zhang Z, Lin QX, Zheng XL, Wu SL, 720 Cheng JD. 2017. CircRNA_000203 enhances the expression of fibrosis-associated genes by 721 derepressing targets of miR-26b-5p, Colla 2 and CTGF, in cardiac fibroblasts. Scientific 722 Reports 7:40342.

723 Thomson DW, Dinger ME. 2016. Endogenous microRNA sponges: evidence and controversy. Nature Reviews Genetics 17: 272-283 DOI: 10.1038/nrg.2016.20

Thum T, Condorelli G. 2015. Long noncoding RNAs and microRNAs in cardiovascular pathophysiology. Circulation Research 116:751-762 DOI: 10.1161/

\section{CIRCRESAHA.116.303549.}

Uchida S, Dimmeler S. 2015. Long noncoding RNAs in cardiovascular diseases. Circulation Research 116:737-750 DOI: 10.1161/CIRCRESAHA.116.302521.

730

731

Vausort M, Salgado-Somoza A, Zhang L, Leszek P, Scholz M, Teren A, Burkhardt R, Thiery J,

732 Wagner DR, Devaux Y. 2016. Myocardial infarction-associated circular RNA predicting left ventricular dysfunction. Journal of the American College of Cardiology 68:1247-1248.

734 Vegter EL, van der Meer P, de Windt LJ, Pinto YM, Voors AA. 2016. MicroRNAs in heart failure: from biomarker to target for therapy. European Journal of Heart Failure 18: 457-468 DOI: $10.1002 /$ ejhf.495.

Wang J, Chen J, Sen S. 2016. MicroRNA as biomarkers and diagnostics. Journal of Cellular

737

738 Physiology 231:25-30 DOI: 10.1002/jcp.25056.

739

Wang K, Gan TY, Li N, Liu CY, Zhou LY, Gao JN. 2017. Circular RNA mediates

741

742

743

744

745

746

747

748

749

750

751 cardiomyocyte death via miRNA-dependent upregulation of MTP18 expression. Cell Death \& Differentiation 24:1111-1120.

Wang K, Long B, Liu F, Wang JX, Liu CY, Zhao B, Zhou LY, Sun T, Wang M, Yu T, Gong Y. 2016. A circular RNA protects the heart from pathological hypertrophy and heart failure by targeting miR-223. European Heart Journal 37:2602-2611.

Wang K, Sun Y, Tao W, Fei X, Chang C. 2017. Androgen receptor (AR) promotes clear cell renal cell carcinoma (ccRCC) migration and invasion via altering the circHIAT1/miR-1955p/29a-3p/29c-3p/CDC42 signals. Cancer Letters 394:1-12.

Wang PL, Bao Y, Yee MC, Barrett SP, Hogan GJ, Olsen MN, Dinneny JR, Brown PO, Salzman J. 2014. Circular RNA is expressed across the eukaryotic tree of life. PLoS One 9:e90859.

Wang Y, Sui X, Zhao H, Cong L, Li Y, Xin T, Guo M, Hao W. 2018. Decreased circular RNA hsa_circ_0001649 predicts unfavorable prognosis in glioma and exerts oncogenic properties in vitro and in vivo. Gene 676, 117-122 DOI: 10.1016/j.gene.2018.07.037.

Peer) reviewing PDF | (2019:01:34077:2:0:NEW 16 Mar 2019) 
752

753

754

755

756

757

758

759

760

761

762

763

764

765

766

767

768

769

770

771

772

773

774

775

776

777

778

779

780

781

782

783

784

785

786

787

788

789

790

Wellcome Trust Case Control Consortium. 2007. Genome-wide association study of 14,000 cases of seven common diseases and 3,000 shared controls. Nature 447:661-678.

Wilusz JE, Sharp PA. 2013. A circuitous route to noncoding RNA. Science 340:440-441 (2013).

Wojciechowska A, Braniewska A, Kozar-Kamińska K. 2017. MicroRNA in cardiovascular biology and disease. Advances in Clinical and Experimental Medicine 26:865-874.

Wu GC, Li J, Leng RX, Li XP, Li XM, Wang DG, Pan HF, Ye DQ. 2017. Identification of long non-coding RNAs GAS5, linc0597 and lnc-DC in plasma as novel biomarkers for systemic lupus erythematosus. Oncotarget 8:23650-23663 DOI: 10.18632/oncotarget.15569.

Wu N, Jin L, Cai J. 2017. Profiling and bioinformatics analyses reveal differential circular RNA expression in hypertensive patients. Clinical and Experimental Hypertension 39:454-459.

Xia L, Song M, Sun M, Wang F, Yang C. 2018. Circular RNAs as biomarkers for cancer. Advances in Experimental Medicine and Biology 1087:171-187 DOI: 10.1007/978-981-131426-1_14.

Xie YZ, Yang F, Tan W, Li X, Jiao C, Huang R, Yang BB. 2016. The anti-cancer components of Ganoderma lucidum possesses cardiovascular protective effect by regulating circular RNA expression. Oncoscience 3:203-207. https://doi.org/10.18632/oncoscience.316

Xing L, Zhang L, Feng Y, Cui Z, Ding L. 2018. Downregulation of circular RNA hsa_circ_0001649 indicates poor prognosis for retinoblastoma and regulates cell proliferation and apoptosis via AKT/mTOR signaling pathway. Biomedicine \& Pharmacotherapy 105:326-333 DOI: 10.1016/j.biopha.2018.05.141.

Xu L, Zhang M, Zheng X, Yi P, Lan C, Xu M. 2017. The circular RNA ciRS-7 (Cdrlas) acts as a risk factor of hepatic microvascular invasion in hepatocellular carcinoma. Journal of Cancer Research and Clinical Oncology 143:17-27.

Yang Y, Fan X, Mao M, Song X, Wu P, Zhang Y, Jin Y, Yang Y, Chen LL, Wang Y, Wong CC. 2017. Extensive translation of circular RNAs driven by N6-methyladenosine. Cell Research 27:626-641.

Yang Y, Gao X, Zhang M, Yan S, Sun C, Xiao F, Huang N, Yang X, Zhao K, Zhou H, Huang S. 2018. Novel role of FBXW7 circular RNA in repressing glioma tumorigenesis. Journal of the National Cancer Institute 110.

Yang Y, Yu T, Jiang S, Zhang Y, Li M, Tang N, Ponnusamy M, Wang JX, Li P. 2017. miRNAs as potential therapeutic targets and diagnostic biomarkers for cardiovascular disease with a particular focus on WO2010091204. Expert Opinion on Therapeutic Patents 27: 1021-1029 DOI: 10.1080/13543776.2017.1344217.

You X, Vlatkovic I, Babic A, Will T, Epstein I, Tushev G, Akbalik G, Wang M, Glock C, Quedenau C, Wang X. 2015. Neural circular RNAs are derived from syn-aptic genes and regulated by development and plasticity. Nature Neuroscience 18:603-610.

Zhang X, Zhou H, Jing W, Luo P, Qiu S, Liu X, Zhu M, Liang C, Yu M, Tu J. 2018. The circular RNA hsa_circ_0001445 regulates the proliferation and migration of hepatocellular 
791

792

793

794

795

796

797

798

799

800

801

802

803

804

805

806

807

808

809

810

811

812

813

814

815

816

817

818

819

820

821

822

823

824

825

826

827

828

829

830

carcinoma and may serve as a diagnostic biomarker. Disease Markers 2018:3073467 DOI: $10.1155 / 2018 / 3073467$.

Zhang XO, Dong R, Zhang Y, Zhang JL, Luo Z, Zhang J, Chen LL, Yang L. 2016. Diverse alternative back-splicing and alternative splicing landscape of circular RNAs. Genome Research 26:1277-1287 DOI: 10.1101/gr.202895.115.

Zhang Y, Liang W, Zhang P, Chen J, Qian H, Zhang X, Xu W. 2017. Circular RNAs: emerging cancer biomarkers and targets. Journal of Experimental \& Clinical Cancer Research 36:152 DOI: 10.1186/s13046-017-0624-z. Review

Zhang Y, Liu B, Shao C, Xu H, Xue A, Zhao Z, Shen Y, Tang Q, Xie J. 2018. Evaluation of the inclusion of circular RNAs in mRNA profiling in forensic body fluid identification. International Journal of Legal Medicine 132:43-52 DOI: 10.1007/s00414-017-1690-7.

Zhang Y, Zhang XO, Chen T, Xiang JF, Yin QF, Xing YH, Zhu S, Yang L, Chen LL. 2013. Circular intronic long noncoding RNAs. Molecular Cell 51:792-806.

Zhao J, Tao Y, Zhou Y, Qin N, Chen C, Tian D, Xu L. 2015. MicroRNA- 7: a promising new target in cancer therapy. Cancer Cell International 15:103 DOI: 10.1186/s12935-015-0259-0

Zhao Z, Li X, Gao C, Jian D, Hao P, Rao L, Li M. 2017. Peripheral blood circular RNA hsa_circ_0124644 can be used as a diagnostic biomarker of coronary artery disease. Scientific Reports 7:39918.

Zeng Y, Du WW, Wu Y, Yang Z, Awan FM, Li X, Yang W, Zhang C, Yang Q, Yee A, Chen Y. 2017. A circular RNA binds to and activates AKT phosphorylation and nuclear localization reducing apoptosis and enhancing cardiac repair. Theranostics 7:3842-3855.

Zheng Q, Bao C, Guo W, Li S, Chen J, Chen B, Luo Y, Lyu D, Li Y, Shi G, Liang L. 2016. Circular RNA profiling reveals an abundant circHIPK3 that regulates cell growth by sponging multiple miRNAs. Nature Communications 7.

Zhong Z, Lv M, Chen J. 2016. Screening differential circular RNA expression profiles reveals the regulatory role of circTCF25-miR-103a-3p/miR-107-CDK6 pathway in bladder carcinoma. Scientific Reports 6:30919.

Zhou B, Jian-Wu Yu JW. 2017. A novel identified circular RNA, circRNA_010567, promotes myocardial fibrosis via suppressing miR-141 by targeting TGF-b1. Biochemical and Biophysical Research Communications 487:769-775.

Zhou LY, Zhai M, Huang Y, Xu S, An T, Wang YH, Zhang RC, Liu CY, Dong YH, Wang M, Qian LL. 2018. The circular RNA ACR attenuates myocardial ischemia/reperfusion injury by suppressing autophagy via modulation of the Pink1/FAM65B pathway. Cell Death \& Differentiation. doi: 10.1038/s41418-018-0206-4.

Zou M, Huang C, Li X, He X, Chen Y, Liao W, Liao Y, Sun J, Liu Z, Zhong L, Bin J. 2017. Circular RNA expression profile and potential function of hsa_circRNA_101238 in human thoracic aortic dissection. Oncotarget 47:81825-81837.

Peer) reviewing PDF | (2019:01:34077:2:0:NEW 16 Mar 2019) 
831

832

833

834

835

836

837

838

839

840

841

842

843

844

845

846

847

848

849

\section{Figure legends}

\section{Figure 1. Percentage of each non-coding RNAs class.}

852 Percentage of the regulatory non-coding RNAs class. Among these non-coding RNAs, long non853 coding RNAs occupy the largest proportion $(69.42 \%)$. The following proportion are snRNAs 854 (8.21\%), Mt_tRNAs (0.095\%), scaRNAs (0.21\%), snoRNAs (4.07\%), misc_RNAs (9.56\%), 855 rRNAs $(0.22 \%)$, miRNAs $(8.13 \%)$, other RNAs including (sRNAs, Mt_rRNAs, ribozyme and 856 vault RNAs) account $0.069 \%$.

\section{Figure 2. The proposed models of circRNA formation}

858 a. Direct lariat-driven cyclization. Exon splicing generates a lariat structure. The 3' splice donor 859 site of exon 1 covalently links to the 5' splice acceptor of exon 4. Circular exonic RNA is formed 860 after removal of intronic sequence. 
861 b. Intron-pairing-driven circularization. Direct base-pairing of the introns flanking inverted 862 repeats or ALU elements leads to the formation of a circular structure. The introns are removed 863 or retained to form exonic circRNA or exon-intron circRNA.

864 c. The circular intronic RNAs are generated from lariat introns that can escape debranching; $7 \mathrm{nt}$ 865 GU-rich sequences near exon 1 (yellow box) and 11 nt C-rich sequences near exon 2 (blue box) 866 form the circular intronic RNAs, by avoiding debranching and become a stable circRNA.

867 d. RNA binding protein (RBP)-driven circularization: circRNA is formed through RBPs (Y868 shape), and introns are removed.

869

870 Figure 3. The circ101238/miR/target gene regulatory network

871 circ101238 negatively regulate gene expression by absorption and sequestration of the five main 872 miRNA molecules.

873 Contributions

874 W.T.W, B.L, H.L.P, M.X.H, and Y.W wrote the paper. Z.C.Z, D.L, T.C.W, R.H.X, and K.X.L

875 checked the References. All authors reviewed the final manuscript.

876

877 Additional Information

878 Competing Interests: The authors declare that they have no competing interests.

879

880 Abbreviation

881 noncoding RNAs (ncRNAs); microRNAs (miRNAs) ;small nuclear RNA (snRNAs); piwi-

882 interacting RNA (piRNAs); small interfering RNA (siRNAs) ; long noncoding RNAs

883 (lncRNAs); cardiovascular disease (CVD); circular RNAs (circRNAs); major adverse

884 cardiovascular events (MACE) ; myocardial infarction (MI); beta-natriuretic peptide (BNP);

885 heart failure (HF); RNA sequencing (RNA-Seq) ; RNA-binding proteins (RBP); Quaking (QKI)

886 ; Muscleblind (MBL) ; adenosine deaminases acting on RNAs (ADAR1); heterogeneous nuclear

887 ribonucleoprotein ( $\mathrm{hnRNP}$ ); serine-arginine (SR); fragile mental retardation protein

888 (FMRP); Argonaute 2 (AGO2); polyadenylate binding protein 1(PABPN1); human antigen R

889 (HuR); ANRIL (antisense non-coding RNA in the INK4 locus) ; ankyrin repeat domain

890 52(ANKRD52); polymerase (Pol); Pescadillo (PES); internal ribosome entry site (IRES); rolling

891 circle amplification (RCA); hepatitis D virus antigen (HDAg) ; coronary artery disease(CAD); 
892 pulmonary arterial hypertension $(\mathrm{PAH})$; troponin $\mathrm{T} / \mathrm{I}(\mathrm{TnT} / \mathrm{I})$; transient receptor potential cation 893 channel subfamily M member 3 (TRPM3); Endothelial-to-mesenchymal transition (EndMT); 894 fibroblast-specific protein-1 (FSP-1); transforming growth factor $\beta$ (TGF- $\beta$ ) ; coronary artery 895 endothelial cells (CAEC); creatine phosphokinase, CPK; heart-related circRNA (HRCR); ARC 896 (apoptosis repressor with CARD domain); myocardial infarction-associated circular RNA 897 (MICRA); essential hypertension (EH); thoracic aortic dissection (TAD) 898

899

900

901 


\section{Figure 1}

Percentage of each non-coding RNAs class

Percentage of the regulatory non-coding RNAs class. Among these non-coding RNAs, long non-coding RNAs occupy the largest proportion (69.42\%). The following proportion are snRNAs (8.21\%), Mt_tRNAs (0.095\%), scaRNAs (0.21\%), snoRNAs $(4.07 \%)$, misc_RNAs (9.56\%), rRNAs $(0.22 \%)$, miRNAs (8.13\%), other RNAs including (sRNAs, Mt_rRNAs, ribozyme and vault RNAs) account $0.069 \%$.

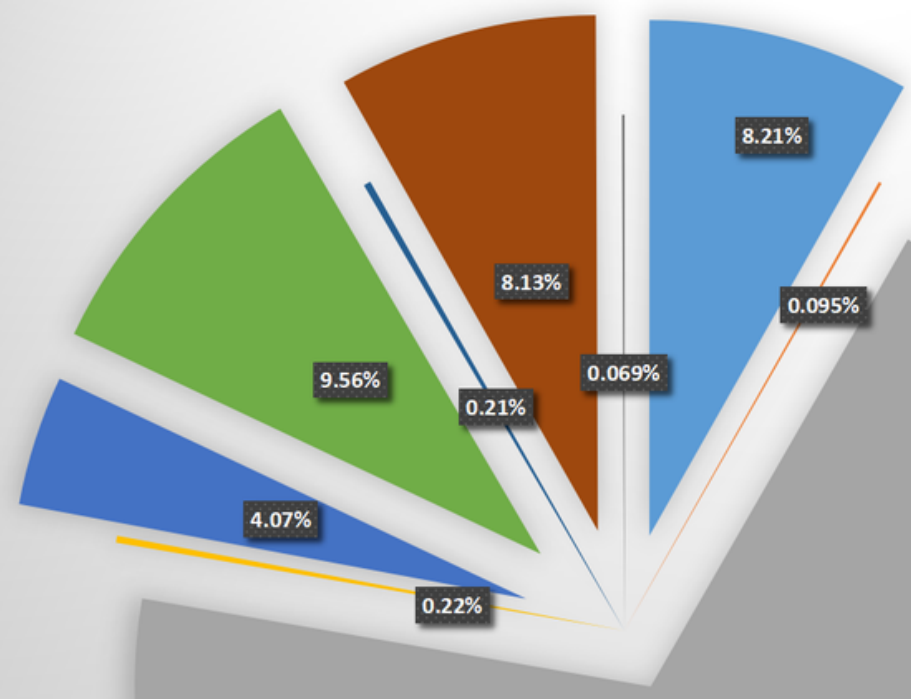




\section{Figure 2}

The proposed models of circRNA formation

a. Direct lariat-driven cyclization. Exon splicing generates a lariat structure. The 3' splice donor site of exon 1 covalently links to the 5 ' splice acceptor of exon 4. Circular exonic RNA is formed after removal of intronic sequence. b. Intron-pairing-driven circularization. Direct base-pairing of the introns flanking inverted repeats or ALU elements leads to the formation of a circular structure. The introns are removed or retained to form exonic circRNA or exonintron circRNA. c. The circular intronic RNAs are generated from lariat introns that can escape debranching; $7 \mathrm{nt} \mathrm{GU}$-rich sequences near exon 1 (yellow box) and $11 \mathrm{nt}$ C-rich sequences near exon 2 (blue box) form the circular intronic RNAs, by avoiding debranching and become a stable circRNA. d. RNA binding protein (RBP)-driven circularization: circRNA is formed through RBPs (Y-shape), and introns are removed. 

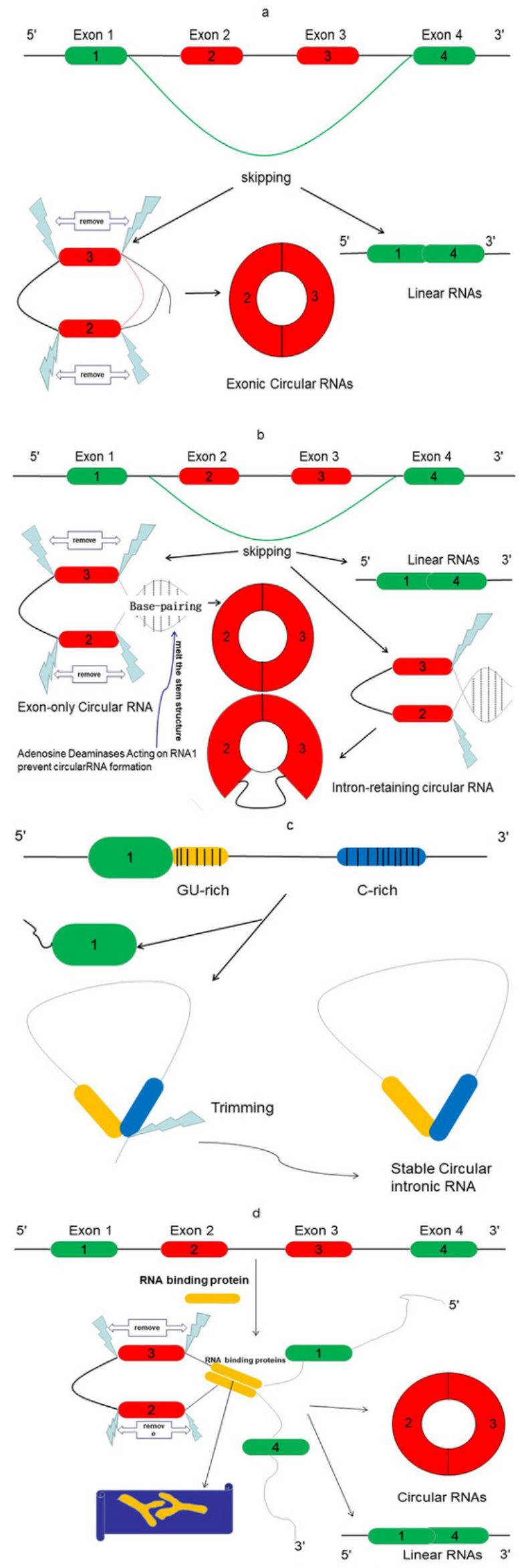

PeerJ reviewing PDF | (2019:01:34077:2:0:NEW 16 Mar 2019) 
Figure 3

\section{The circ101238/miR/target gene regulatory network}

circ101238 negatively regulate gene expression by absorption and sequestration of the five main miRNA molecules.

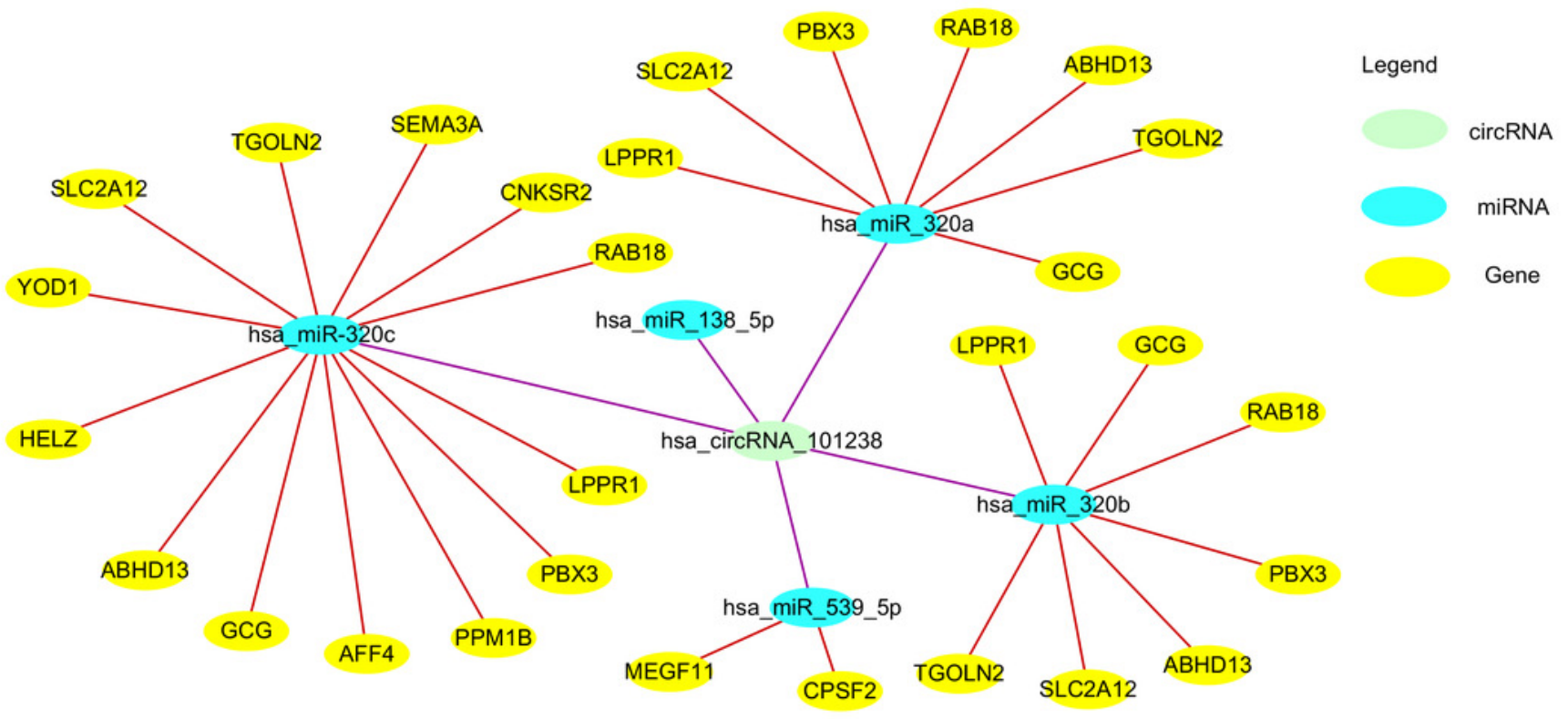

